\title{
Variable Ordering for Efficient SAT Search by Analyzing Constraint-Variable Dependencies
}

\author{
Vijay Durairaj and Priyank Kalla \\ Department of Electrical and Computer Engineering \\ University of Utah, Salt Lake City, UT-84112 \\ \{durairaj, kalla\}@ece.utah.edu
}

\begin{abstract}
This paper presents a new technique to derive an initial static variable ordering for efficient SAT search. Our approach not only exploits variable activity and connectivity information simultaneously, but it also analyzes how tightly the variables are related to each other. For this purpose, a new metric is proposed - the degree of correlation among pairs of variables. Variable activity and correlation information is modeled (implicitly) as a weighted graph. A topological analysis of this graph generates an order for SAT search. An algorithm called ACCORD (ACtivity - CORrelation - ORDering) is proposed for this purpose.

While ACCORD rigorously analyzes constraint-variable dependencies, it does not account for the effect of decision-assignments on clause-variable dependencies. This issue motivates further refinements to our approach using literal activity and correlation measures - giving rise to the L'ACCORD algorithm. Using efficient implementations of the above, experiments are conducted over a wide range of benchmarks. The results demonstrate that: (i) the variable order generated by our approach significantly improves the performance of SAT solvers; (ii) time to derive this order is a fraction of the overall solving time. As a result, our approach delivers faster performance (often, by orders of magnitude) as compared to contemporary approaches.
\end{abstract}

\section{Introduction}

Contemporary SAT solvers have matured over the years and come a long way from the classical search procedures of Davis-Putnam (DP) [1] and Davis-LogemannLoveland (DLL) [2]. Recent approaches [3] [4] [5] etc., employ sophisticated methods such as constraint propagation and simplification, conflict analysis, learning and non-chronological backtracks [3] [4] [5] to efficiently analyze and prune the search space. In recent past, a lot of effort has been invested in trying to understand the nature of the SAT problem. The works that deserve mention relate to symmetry analysis [6] [7], local search strategies [8], complexity of SAT viz-a-viz ATPG [9], relationship of BDD variable orderings and CNF search procedures [10], amount of search space analyzed [11], UNSAT core extraction [12] [13], among others [14] [15] [16].

An important aspect of CNF-SAT is to derive an ordering of variables to guide the search. The order in which variables (and correspondingly, constraints) 
are resolved significantly impacts the performance of SAT search procedures. Boolean functions arising in many applications represent some spatial, casual or logical dependencies (or connections) among variables. Therefore, analysis of these clause-variable dependencies provides useful information that can be exploited to guide CNF-SAT search procedures. Variable activity and clause connectivity are often considered as qualitative and quantitative metrics to model clause-variable dependencies. Activity of a variable (or literal) is defined as the number of its occurrence among all the clauses of a given SAT problem [17]. Most conventional SAT solvers [4] [5] [3] employ variable/literal-activity based branching heuristics to resolve the constraints.

Connectivity of constraints has also been used as a heuristic approach to derive variable orderings for SAT search. Loosely speaking, two clauses are said to be "connected" if one or more variables are common to their support. Clause connectivity can be modeled by representing CNF-SAT constraints as (hyper) graphs and, subsequently, analyzing the graph's topological structure. Tree decomposition techniques have been proposed in literature [18] [19] for analyzing connectivity of constraints in constraint satisfaction programs (CSP). Such techniques identify decompositions with minimum tree-width, thus enabling a partitioning of the overall problem into a chain of connected constraints. Recently, such approaches have also found application in those problems that can be modeled as DPLL-based CNF-SAT search [19] [20] [21] [22] [23] [24]. Various approaches operate on such partitioned tree structures by deriving an order in which the partitioned set of constraints are resolved [20] [21] [22] [24]. MINCE [10] employs CAPO placer's mechanism [25] to find a variable order such that the clauses are resolved according to their chain of connectivity. Bjesse et. al. [21] proposed tree decomposition based approaches to guide variable selection and conflict clause generation. Aloul et. al. have proposed a fast, heuristic based approach, FORCE [26], as an alternative to the computationally complex approach of MINCE. Recently, Durairaj et. al. [27] [28] [29] proposed hypergraph bi-partitioning based constraint decomposition scheme that employs both variable activity and clause connectivity simultaneously to derive a variable order.

This paper presents a new approach to derive an initial static ordering for SAT search by rigorously analyzing constraint-variable dependencies. Experimental results demonstrate that our approach is faster and more robust than the contemporary variable ordering techniques, and it improves the performance of SAT solvers (in many cases by orders of magnitude).

The paper is organized as follows. The following Section analyzes the limitation of previous work. Section 3 outlines the specific contributions of this research. The variable order generation approaches are presented in Sections 4 and 5 , followed by experimental results and analysis in Section 6 . Finally, Section 7 concludes the paper. 


\section{Limitations of Previous Work}

The computational complexity (exponential) of minimum tree-width decomposition algorithms results in large compute times to search for the variable order. The technique of Dechter et. al. [18] is shown to be time exponential in the treewidth. Algorithms for approximating tree-width with bounded error [23] are also shown to be too costly for industrial problems [21]. As a result, these techniques are impractical for large/hard CNF-SAT problems.

In general, the time required to derive a variable order should be small as compared to the subsequent SAT solving time - it should certainly not exceed the solving time. Unfortunately, for large and hard SAT problems, it has been observed that MINCE [10] [30] and Amir's tool [20] [31] require unacceptably long time just to derive the variable order. This behaviour is depicted in Table 1 , which compares the time required to derive the variable order by Amir's tool [31], MINCE [30], FORCE [26] and the hypergraph partitioning based technique (HGPart) of [27] against zCHAFF's (2003 version) solve time. It can be observed from the table that in order to derive the variable order, both Amir's and MINCE approach suffer from long compute times - much longer than the default SAT solving time. In contrast, FORCE and HGPart can derive the variable order much faster than the other two. This clearly demonstrates the computational limitations of Amir's and MINCE approach; as such they are too expensive to be applicable for practical SAT problems. While, FORCE is fast, the quality of the variable order does not consistently improve the performance of SAT solvers.

Table 1. Comparison of original zCHAFF runtime, Amir's, MINCE's, FORCE's and HGPart's partitioning time

\begin{tabular}{||l|c|c|c|c|c|c||}
\hline $\begin{array}{l}\text { Benc- } \\
\text { hmark }\end{array}$ & $\begin{array}{c}\text { Vars/ } \\
\text { Clauses }\end{array}$ & $\begin{array}{c}\text { zCHAFF'03 } \\
(\mathrm{sec})\end{array}$ & $\begin{array}{c}\text { Amir } \\
(\mathrm{sec})\end{array}$ & $\begin{array}{c}\text { MINCE } \\
(\mathrm{sec})\end{array}$ & $\begin{array}{c}\text { FORCE } \\
(\mathrm{sec})\end{array}$ & $\begin{array}{c}\text { HGPart } \\
(\mathrm{sec})\end{array}$ \\
\hline c2670 & $2.5 \mathrm{~K} / 6.4 \mathrm{~K}$ & 1.48 & 16.54 & 8.23 & 0.94 & 1.15 \\
\hline c3540 & $3.4 \mathrm{~K} / 9.2 \mathrm{~K}$ & 20.57 & 23.66 & 13.95 & 0.83 & 1.42 \\
\hline c5315 & $5.0 \mathrm{~K} / 14.1 \mathrm{~K}$ & 34.62 & 54.56 & 25.51 & 6.52 & 1.25 \\
\hline c7552 & $5.5 \mathrm{~K} / 15.1 \mathrm{~K}$ & 105.97 & 71.30 & 24.43 & 3.81 & 1.76 \\
\hline \hline 4pipe & $5.2 \mathrm{~K} / 80.2 \mathrm{~K}$ & 129 & 505.83 & 93.1 & 1.36 & 13.84 \\
\hline 5pipe & $9.5 \mathrm{~K} / 195 \mathrm{~K}$ & 196.53 & $>2000$ & 267.2 & 2.82 & 38.40 \\
\hline
\end{tabular}

Table 2. Quality of variable order derived by FORCE

\begin{tabular}{||l|c|c|c|}
\hline $\begin{array}{l}\text { Benc- } \\
\text { hmark }\end{array}$ & $\begin{array}{c}\text { Vars/ } \\
\text { Clauses }\end{array}$ & $\begin{array}{c}\text { zCHAFF'03 } \\
(\mathrm{sec})\end{array}$ & $\begin{array}{c}\text { FORCE } \\
\text { (sec) }\end{array}$ \\
\hline c3540 & $3431 / 9262$ & $\mathbf{2 0 . 5 7}$ & 36.55 \\
\hline c5315 & $4992 / 14151$ & 34.62 & $\mathbf{9 . 2 5}$ \\
\hline c7552 & $5466 / 15150$ & $\mathbf{1 0 5 . 9 7}$ & 136.55 \\
\hline \hline 4pipe_q0_k & $5380 / 69072$ & $\mathbf{6 6 . 7 1}$ & 87.78 \\
\hline 5pipe_q0_k & $10026 / 154409$ & 649.9 & $\mathbf{3 7 5 . 3 7}$ \\
\hline \hline clus_set1 & $1200 / 4800$ & $\mathbf{5 9 . 6 2}$ & $>1000$ \\
\hline
\end{tabular}


Table 2 presents some results that reflect the inconsistency in the quality of the variable order derived by FORCE. Since MINCE and Amir's tool have been shown to be impractical (too slow), we analyze the variable order derived by FORCE. The order derived by FORCE was given to the zCHAFF solver (2003 version) to resolve constraints - the runtime is given in Column 4. Column 3 corresponds to original zCHAFF runtime (VSIDS heuristic). From the results, it can be observed that the the variable order generated by FORCE does not consistently enhance the performance of the SAT solver ${ }^{1}$. In fact, for some benchmarks, it degrades the SAT solver performance by an unacceptably long time.

Recent approach of [27] has shown to deliver good results. Not only can the variable order be derived in a reasonable amount of time, the quality of the order also consistently improves the performance of the solver. However, as this technique relies on hypergraph partitioning, there is no direct control over the decomposition. As a result, many problems (such as the NQueens) cannot be decomposed efficiently. Another limitation of these techniques is that while they do analyze variable activity, clause connectivity or both, however, they do not analyze how tightly the variables are connected to each other. As a result, tightly connected, hard problems may not realize the run-time improvements.

\section{Research Contributions}

As discussed earlier, efficient CNF-SAT decision heuristics should analyze both variable activity and connectivity simultaneously for faster constraint resolution. Moreover, they should also analyze how tightly the variable are related to each other. Furthermore, the procedure to generate such an order should be fast, scalable and robust enough to handle large SAT problems. This paper proposes an efficient variable order generation procedure that attempts to fulfill the above criteria/requirements.

The contributions of our work are as follows:

1. We present an efficient technique that analyzes constraint-variable dependencies to derive an ordering of variables to guide SAT diagnosis. Both variable activity and connectivity information is exploited to derive the order.

2. In order to analyze how tightly two or more constraints are related (in terms of common variables), we propose a new metric called the degree of correlation among pairs of variables.

3. Variable activity and correlation information is (implicitly) modeled as a weighted graph, the topological analysis of which enables the derivation of such an order. An efficient algorithm (ACCORD) is described for this purpose.

4. In order to analyze the effect of decision-assignments made by SAT solver on the variable ordering, further refinements to the ACCORD algorithm are proposed.

\footnotetext{
${ }^{1}$ Mince also exhibits similar phenomenon.
} 
5. Experiments conducted over a large and varied set of benchmarks demonstrate that our approach is fast, robust and scalable; quality of the variable order is reflected in the impressive speed-up achieved for SAT solving. Particularly for hard instances, orders of magnitude speed-up is achieved in many cases.

6. The variable order generation time is small as compared to the overall solving time.

\section{Variable Order Computation: Analyzing Constraint-Variable Dependencies}

It is our desire to derive a variable order for SAT search by analyzing clausevariable relationships. The importance of branching on high activity variables is well understood [17]. Analyzing the connectivity of constraints is also important for constraint resolution. Contemporary techniques address both of the above issues. However, 'how tightly are the variables related?' - this feature too should not be overlooked. For this purpose, we propose a metric that measures how tightly the variables are connected/related. We define this metric as follows:

Definition 41 Two variables $x_{i}$ and $x_{j}$ are said to be correlated if they appear together (as literals) in one or more clauses. The number of clauses in which the pair of variables $\left(x_{i}, x_{j}\right)$ appear together is termed as their degree of correlation.

\subsection{Problem Modeling}

In our approach, the constraint-variable relationship of a given CNF-SAT problem is modeled as a weighted graph. The variables (as opposed to literals) form the vertices, while edges denote the correlation/connectivity between them. Associated with each variable is its activity, which is modeled as an integer value within the node. The edge weights represent the degree of correlation between the variables/vertices. For example, if two variables $x_{i}, x_{j}$ appear together in $n$ clauses, then the weight of the edge $e_{i j}$ connecting them is $n$.

An ordering of the nodes (variable order) can be performed by analyzing the graph's topology. We now describe our approach by means of an example corresponding to the CNF-SAT problem shown in Fig. 1. Its corresponding weighted graph is depicted in Fig. 2(a).

\subsection{ACCORD: Activity-Correlation based Ordering}

We begin the search by first selecting the highest active variable, i.e. the node that has the highest internal weight. The variable is marked and the node is added to a set called supernode. The variable is also stored in a list (var_ord_list). The SAT tool should branch on this variable first. It can be observed from the graph (weight within the nodes) that the activity of variables $\{e, u, g, v\}$ is the 


$$
\begin{aligned}
& (a+b+\bar{u}) \\
& (\bar{a}+u)(\bar{b}+u) \\
& (u+g)(c+g) \\
& (\bar{u}+\bar{c}+g) \\
& (a+\bar{e})(\bar{b}+\bar{e}) \\
& (a+b+e) \\
& (v+d+\bar{w}) \\
& (\bar{v}+w)(d+w) \\
& (e+g+\bar{v}) \\
& (e+v)(\bar{g}+v)
\end{aligned}
$$

Fig. 1. An example CNF-SAT problem

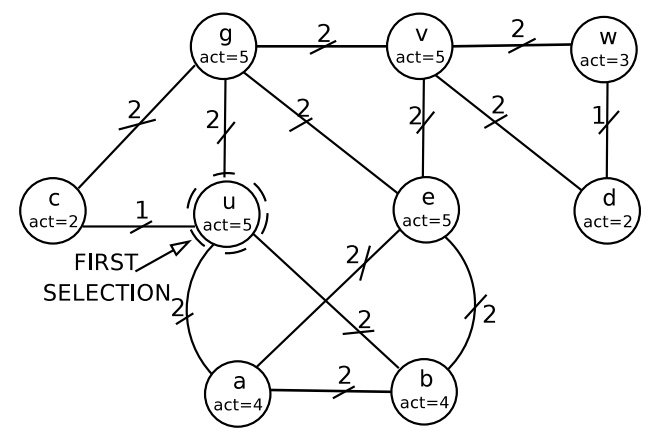

(a)

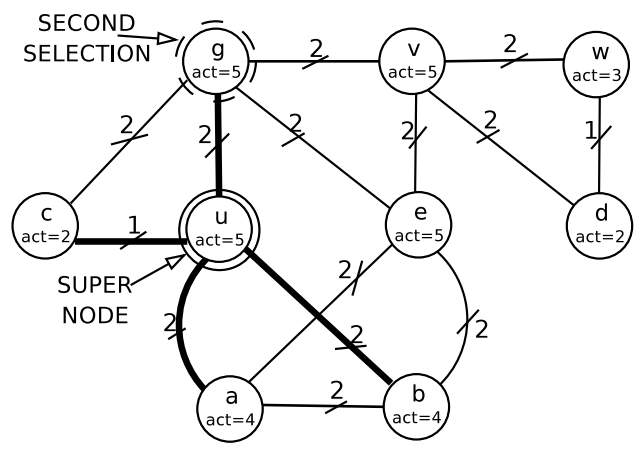

(b)

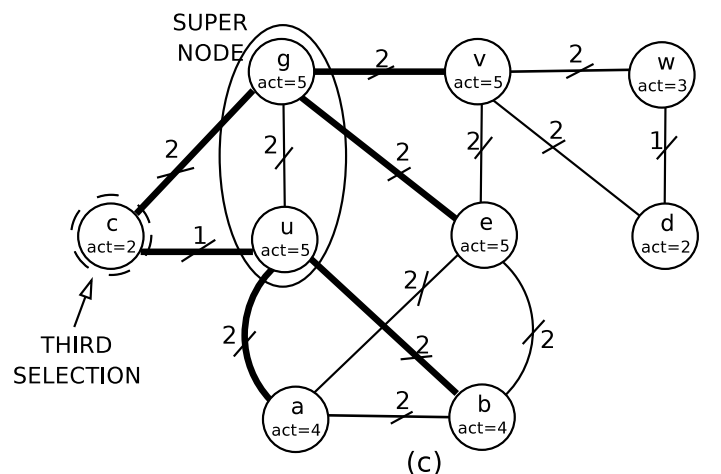

Fig. 2. Weighted Graph - Edge weights denote the degree of correlation between the variables (vertices). Variable activity depicted within the node. 
highest $(=5)$. We select one of these variables (randomly or lexicographically) as the initial branching variable. Let us select the variable $u$, shown in Fig. 2(a) as "First Selection." The variable $u$ becomes the supernode.

Now we need to identify the set of variables connected to this supernode. All the nodes that share at least one edge with the supernode are identified. The nodes $\{g, c, a, b\}$ are connected to the supernode $u$, as shown in Fig. 2(b). One of these variables $\{g, c, a, b\}$ is to be selected as the next branching variable. We consider the degree of correlation, modeled as edge weights, as the metric to identify this variable. The nodes that share an edge with the supernode are sorted in decreasing order of the sum of their degrees of correlation with the nodes in the supernode. In our example, variable $c$ has the degree of correlation $=1$. On the other hand, the variables $\{g, a, b\}$ have the same degree of correlation with node $u$, which is equal to 2 ; hence, we consider variables $\{g, a, b\}$. In order to break this tie, we further distinguish these variables according to their activity. Since the activity of variable $g$ is higher than that of variables $\{a, b\}, g$ is selected as the next branching variable. This is shown in Fig. 2(b) as "Second Selection." Moreover, the node $g$ is added to the current supernode set; supernode $=\{u, g\}$. The variable order derived so far is var_ord_list $=\{u, g\}$.

As the next step, those variables are identified that share an edge with the supernode. These variables are $\{v, e, c, a, b\}$ as shown in Fig.2(c) with highlighted incident edges. Consider the nodes $\{v, e, a, b\}$. They share only one edge with the supernode with weights (correlation) $=2$. On the other hand, node $c$ has two edges incident on the supernode. Therefore, the correlation modeled by both edges should be accounted for. This is computed as the sum of the degrees of correlation between $c$ and the supernode $\{g, u\}=2+1=3$. Since this sum is the highest for $c$, it is selected as the next branching variable. This is shown in Fig. 2(c) as third selection. The supernode set and the var_ord_list are correspondingly updated. The above procedure is repeated until all the nodes are ordered and included with in the supernode. The final derived variable order is $\{u, g, c, v, e, a, b, w, d\}$.

Our approach is inspired from the Prim's Minimum Spanning Tree (MST) algorithm [32]. We have named it the ACCORD (ACtivity COrrelation ORDering) algorithm. The Pseudo code for the ACCORD algorithm is presented in Algorithm 1. The Variable-Clause database, mentioned in lines 1-3, is implemented using the array of arrays data-structure available within contemporary SAT solvers [4] [33]. For example, the Variable-Clause database corresponding to the CNF-SAT problem of Fig 1 is shown in Fig. 3. The variable $a$ appears in Clauses $\{1,2,7,9\}$. Hence, the first element of Variable-Clause database array is an array containing $\{1,2,7,9\}$. Similarly, the second element of the array is an array containing $\{1,3,8,9\}$ corresponding to variable $b$; and so on. The algorithm analyzes the clause-variable database, and for each variable, it computes its correlated variables. Using the activity and correlation measures, the variable ordering is computed. 

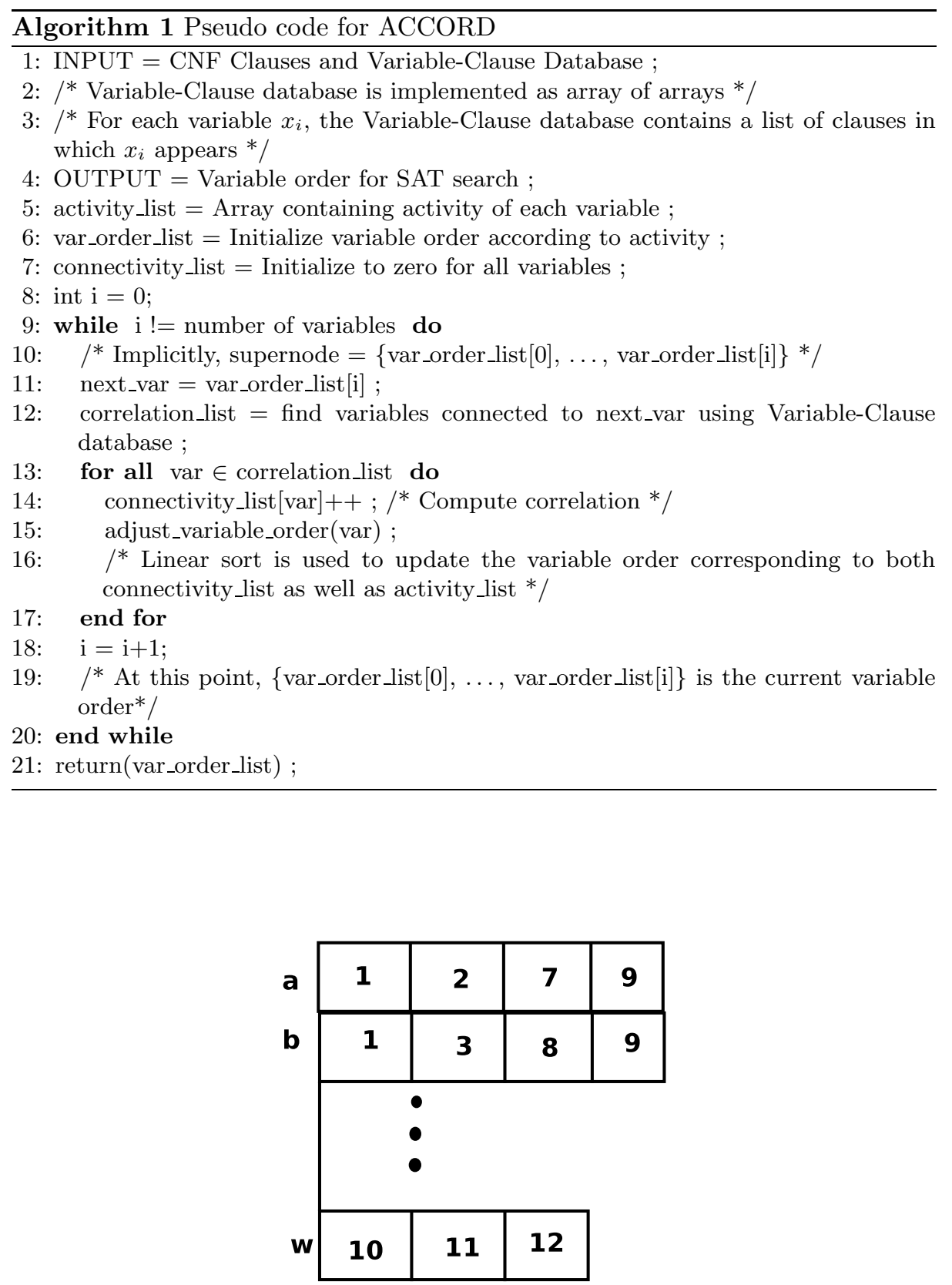

Fig. 3. Variable-Clause Database 


\subsection{Complexity of ACCORD}

A weak upper bound for the ACCORD algorithm can be found by assuming that every variable appears in every other clause. With this assumption, an upper bound on the time complexity of ACCORD can be derived as $O\left(V \cdot\left(V \cdot C+V^{2}\right)\right)$, where $V$ represents the number of variables and $C$ represents the number of clauses.

\section{L'ACCORD: Overcoming the Limitations of ACCORD}

Most conventional SAT solvers employ literal-activity based branching heuristics to resolve the constraints. Also, when the variable order generated by ACCORD is given to a SAT solver, the solver will branch on a literal corresponding to the variable selected by the ACCORD. Clauses corresponding to this literal will be satisfied due to this assignment. ACCORD does not analyze this effect of decision-assignments on the variable correlation. Consider the following set of clauses.

$$
\begin{gathered}
(x+a+e)(x+b+f)(x+c+\bar{e}) \\
(\bar{x}+d+f)(\bar{x}+c)
\end{gathered}
$$

Here, the literal $x$ has the highest activity and the SAT solver will assign $x=1$. This satisfies the first three clauses. The ACCORD algorithm will consider the variables $\{\mathrm{a}, \mathrm{e}, \mathrm{b}, \mathrm{f}, \mathrm{c}, \mathrm{d}\}$ to compute their respective degrees of correlation with respect to $x$. However, due to the assignment $x=1$, it should suffice to look at only those clauses in which $\bar{x}$ appears, as they are unsatisfied. This means that the correlated variables to $\bar{x}(\{\mathrm{~d}, \mathrm{f}, \mathrm{c}\})$ and their corresponding constraints should be analyzed to derive a variable order. In order to exploit such a behaviour, we have implemented the above modifications to the ACCORD algorithm. Instead of analyzing variable-to-variable correlations, we now analyze correlations between a literal and its connected variables. The modified algorithm is now called L' ACCORD.

\section{$6 \quad$ Experimental Results and Analysis}

The ACCORD and L' ACCORD algorithms have been programmed within the zCHAFF [4] solver using its native data-structures. The algorithms analyze the constraint-variable relationships of the given CNF-SAT problem and derive a variable order for SAT search. Using this as the initial order, the SAT tool (zCHAFF) performs a search for the solutions. On encountering conflicts, we allow the solver to add conflict-induced clauses and proceed with its book-keeping and (non-chronological) backtracking procedures. In other words, ACCORD/L' ACCORD provide only an initial static ordering. zCHAFF's VSIDS heuristic updates this order dynamically, when conflict clauses are added. Hence, our approach is not a replacement for VSIDS; it is to be used in conjunction with it. 
Using this setup, we have conducted experiments over a large set of benchmarks that include: i) Microprocessor verification benchmarks [34]; and ii) some of the hard instances specifically created for the SAT competition (all three categories - industrial, handmade and random). We conducted our experiments on a Linux workstation with a $2.6 \mathrm{GHz}$ Pentium-IV processor and 512MB RAM.

\subsection{ACCORD versus L' ACCORD comparison}

Table 3 compares the quality of orders generated by ACCORD and L' ACCORD. It is clear from the table that L' ACCORD generates a better quality variable order than ACCORD, always improving the SAT solver performance.

Table 3. Run-time Comparison of ACCORD and L' ACCORD

\begin{tabular}{|c|c|c|c|c|c|c|c|}
\hline & zCHAFF & \multicolumn{3}{|c|}{ ACCORD + zCHAFF } & \multicolumn{3}{|c|}{ L' ACCORD + zCHAFF } \\
\hline $\begin{array}{l}\text { Bench- } \\
\text { mark }\end{array}$ & $\begin{array}{l}\text { Solve } \\
(\mathrm{sec})\end{array}$ & $\begin{array}{c}\text { Var. } \\
\text { Time(s) }\end{array}$ & $\begin{array}{c}\text { Solve } \\
\text { Time(s) }\end{array}$ & \begin{tabular}{|c|} 
Total \\
Time $(\mathrm{s})$
\end{tabular} & $\begin{array}{c}\text { Var. } \\
\text { Time(s) }\end{array}$ & \begin{tabular}{|c|} 
Solve \\
Time(s)
\end{tabular} & \begin{tabular}{|c|} 
Total \\
Time(s)
\end{tabular} \\
\hline clus-2020-1 & $>1000$ & 0.01 & 746.62 & 746.63 & 0.01 & 666.32 & 666.33 \\
\hline clus-1010-3 & $>1000$ & 0.01 & $>1000$ & - & 0.01 & 700.85 & 700.86 \\
\hline conn-n600-939 & 135.54 & 0.01 & 82.82 & 82.83 & 0 & 0.42 & 0.42 \\
\hline conn-n600-945 & 581.56 & 0.01 & 534.64 & 534.65 & 0.01 & 98.02 & 98.03 \\
\hline icos-stretch & 102.99 & 0 & 111.39 & 111.39 & 0 & 78.56 & 78.56 \\
\hline marg-33-add8ch & 247.73 & 0 & 21.8 & 21.8 & 0 & 20.01 & 20.01 \\
\hline marg-35-1452 & $>1000$ & 0 & $>1000$ & - & 0 & 289.17 & 289.17 \\
\hline mm-1x10-1488 & 654.16 & 0.01 & $>1000$ & - & 0.01 & 237.48 & 237.49 \\
\hline mm-2x2-50-149 & 39.8 & 0.02 & 27.64 & 27.66 & 0.05 & 24.9 & 24.95 \\
\hline qwh-35-405 & 39.73 & 0.01 & 114.45 & 114.46 & 0.03 & 5.58 & 5.61 \\
\hline urqh1c3x3 & 284.48 & 0 & 8.53 & 8.53 & 0 & 57.96 & 57.96 \\
\hline urqh2x 4 & 302.79 & 0 & 33.39 & 33.39 & 0 & 26.53 & 26.53 \\
\hline urqh $2 \times 5$ & $>1000$ & 0 & $>1000$ & - & 0 & 612.96 & 612.96 \\
\hline urqh1c3x4 & $>1000$ & 0 & $>1000$ & - & 0 & 320.62 & 320.62 \\
\hline unif-r4-2 & $>>1000$ & $\overline{\overline{0}}$ & $>>1000$ & - & 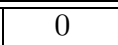 & 198.47 & 198.47 \\
\hline unif-r4-7 & $>1000$ & 0 & 515.24 & 515.241 & 0 & \begin{tabular}{|l|}
151.25 \\
\end{tabular} & 151.25 \\
\hline unif-r4-9 & $>1000$ & 0 & 350.12 & 350.12 & 0 & 141.95 & 141.95 \\
\hline 3bitadd_31 & 68.88 & 0.14 & 43.03 & 43.17 & 0.09 & 4.1 & 4.19 \\
\hline 3bitadd_32 & 7.43 & 0.15 & 0.86 & 1.01 & 0.1 & 0.02 & 0.12 \\
\hline 9dlx-vliw-iq1 & 504.47 & 12.79 & 464.85 & 477.64 & 15.69 & \begin{tabular}{|l|}
381.09 \\
\end{tabular} & \begin{tabular}{|l|}
396.78 \\
\end{tabular} \\
\hline
\end{tabular}

\subsection{L' ACCORD versus Contemporary Techniques}

Table 4 presents some results that compares the quality of the variable order derived by L' ACCORD with those of zCHAFF (latest version developed in 2004) and hypergraph partitioning (HGPart) based order [27]. For a fair comparison, zCHAFF is used as the base SAT solver for all experiments - just the 
variable orders are different. As compared to zCHAFF and HGPart, the variable order generated by L' ACCORD results in a orders of magnitude speedup in SAT solving. Particularly for more difficult problems, our approach significantly outperforms the other two.

Table 4. Run-time Comparison of L' ACCORD with zCHAFF and Hypergraph Partitioning based Technique

\begin{tabular}{|c|c|c|c|c|c|c|c|c|c|}
\hline & & & zCHAFF & \multicolumn{3}{|c|}{ HGPart + zCHAFF } & \multicolumn{3}{|c|}{ L' ACCORD + zCHAFF } \\
\hline $\begin{array}{l}\text { Bench- } \\
\text { mark }\end{array}$ & $\begin{array}{c}\text { Vars/ } \\
\text { Clauses }\end{array}$ & $\begin{array}{c}\text { SAT/ } \\
\text { UNSAT }\end{array}$ & $\begin{array}{l}\text { Solve } \\
(\mathrm{sec})\end{array}$ & \begin{tabular}{|c|} 
Var. \\
Time(s)
\end{tabular} & \begin{tabular}{|c} 
Solve \\
Time(s)
\end{tabular} & \begin{tabular}{|c|} 
Total \\
Time $(\mathrm{s})$
\end{tabular} & $\begin{array}{c}\text { Var. } \\
\text { Time(s) }\end{array}$ & $\begin{array}{c}\text { Solve } \\
\text { Time(s) }\end{array}$ & $\begin{array}{c}\text { Total } \\
\text { Time }(\mathrm{s})\end{array}$ \\
\hline 3bitadd_31 & $8432 / 21210$ & $\mathrm{~S}$ & 68.88 & 3.023 & 0.55 & 3.573 & 0.09 & 4.1 & 4.19 \\
\hline 3bitadd_32 & $8704 / 32316$ & $\mathrm{~S}$ & 7.43 & 3.417 & 0.67 & 4.087 & 0.1 & 0.02 & 0.12 \\
\hline clus-2020-1 & $1200 / 4800$ & $\mathrm{~S}$ & $>1000$ & 1.421 & $>1000$ & - & 0.01 & 666.32 & 666.33 \\
\hline clus-2020-2 & $1200 / 4800$ & $\mathrm{~S}$ & 688.19 & 1.81 & 9.12 & 10.93 & 0.04 & 37.17 & 37.21 \\
\hline clus-4530-2 & $1200 / 4800$ & $\mathrm{~S}$ & 975.83 & 1.846 & 43.85 & 45.696 & 0.02 & 630.55 & 630.57 \\
\hline clus-1010-3 & $1200 / 4919$ & $\mathrm{~S}$ & $>1000$ & 1.701 & 704.66 & 706.361 & 0.01 & 700.85 & 700.86 \\
\hline color-10-3 & $300 / 6475$ & $\mathrm{~S}$ & 129.57 & 0.375 & 19.01 & 19.385 & 0 & 25.05 & 25.05 \\
\hline conn-n600-939 & $576 / 6864$ & $\mathrm{~S}$ & 135.54 & 1.196 & 28.96 & 30.156 & 0 & 0.42 & 0.42 \\
\hline conn-n600-945 & $596 / 7157$ & $\mathrm{~S}$ & 581.56 & 1.145 & $>1000$ & - & 0.01 & 98.02 & 98.03 \\
\hline icos-stretch & $45 / 352$ & $\mathrm{U}$ & 102.99 & 0.199 & 93.84 & 94.039 & 0 & 78.56 & 78.56 \\
\hline marg-33-add8ch & $41 / 272$ & $\mathrm{U}$ & 247.73 & 0.071 & 142.53 & 142.601 & 0 & 20.01 & 20.01 \\
\hline marg-33-add8 & $41 / 224$ & $\mathrm{U}$ & 3.44 & 0.074 & 1.36 & 1.434 & 0 & 1.29 & 1.29 \\
\hline marg-35-1452 & $61 / 280$ & $\mathrm{U}$ & $>1000$ & 0.085 & $>1000$ & - & 0 & 289.17 & 289.17 \\
\hline mm-1x10-1488 & $1120 / 7220$ & $\mathrm{~S}$ & 654.16 & 1.057 & $>1000$ & - & 0.01 & 237.48 & 237.49 \\
\hline mm-2x2-50-1496 & $60 / 32000$ & $\mathrm{U}$ & 39.8 & 3.815 & 31.37 & 35.185 & 0.05 & 24.9 & 24.95 \\
\hline mm-2x3-66-1502 & $1698 / 48771$ & $\mathrm{U}$ & 17.32 & 4.386 & 16.48 & 20.866 & 0.08 & 13.55 & 13.63 \\
\hline qwh-35-405 & $1597 / 10658$ & $\mathrm{~S}$ & 39.73 & 1.869 & 84.59 & 86.459 & 0.03 & 5.58 & 5.61 \\
\hline urqh1c3x3 & $41 / 204$ & $\mathrm{U}$ & 284.48 & 0.078 & 8.92 & 8.998 & 0 & 57.96 & 57.96 \\
\hline urqh2x 4 & $42 / 336$ & $\mathrm{U}$ & 302.79 & 0.077 & 71.07 & 71.147 & 0 & 26.53 & 26.53 \\
\hline urqh2x5 & $53 / 432$ & $\mathrm{U}$ & $>1000$ & 0.117 & $>1000$ & - & 0 & 612.96 & 612.96 \\
\hline urqh1c3x4 & $58 / 476$ & $\mathrm{U}$ & $>1000$ & 0.143 & \begin{tabular}{|l|}
196.78 \\
\end{tabular} & 196.923 & 0 & 320.62 & 320.62 \\
\hline unif-r4-2 & $500 / 2000$ & $\overline{\mathrm{S}}$ & $>1000$ & 0.447 & $>1000$ & - & $\overline{00}$ & 198.47 & 198.47 \\
\hline unif-r4-5 & $500 / 2000$ & $\mathrm{~S}$ & 884.04 & 0.491 & 91.88 & 92.371 & 0 & 129.79 & 129.79 \\
\hline unif-r4-7 & $500 / 2000$ & $\mathrm{~S}$ & $>1000$ & 0.451 & 582.53 & 582.981 & 0 & 151.25 & 151.25 \\
\hline unif-r4-9 & $500 / 2000$ & $\mathrm{~S}$ & $>1000$ & 0.493 & \begin{tabular}{|l|}
711.65 \\
\end{tabular} & \begin{tabular}{|l|}
712.143 \\
\end{tabular} & 0 & 141.95 & 141.95 \\
\hline ferry10 & $292958 / 20791$ & $\overline{\mathrm{S}}$ & 3.54 & 3.549 & 0.73 & 4.279 & 0.04 & 0.07 & 0.11 \\
\hline ferry12 & $4222 / 32199$ & $\mathrm{~S}$ & 238.5 & 8.189 & \begin{tabular}{|l|}
134.78 \\
\end{tabular} & 142.969 & 0.09 & 66.51 & 66.6 \\
\hline rotmul & $5980 / 35229$ & $\mathrm{U}$ & 174.7 & 5.888 & 153.26 & 159.148 & 0.28 & 159.53 & 159.81 \\
\hline 9dlx-vliw-iq1 & $24604 / 261473$ & $\mathrm{U}$ & 504.47 & 68.83 & \begin{tabular}{|l|}
443.79 \\
\end{tabular} & 512.62 & 15.69 & 381.09 & 396.78 \\
\hline
\end{tabular}

The table 5 depicts some results for the microprocessor pipeline verification benchmarks. These experiments were conducted using both 2003 and 2004 versions of the zCHAFF SAT solver. For both experiments, the same variable order derived by L' ACCORD is used. Note that, using the L' ACCORD's order, 
zCHAFF-2003 tool is able to improve the performance significantly. zCHAFF2004 follows upon its earlier versions by implementing the efficient conflict analysis procedures. As a result, these benchmarks can be quickly solved by the 2004 version of the solver and the impact of L' ACCORD is minimal.

Table 5. Run-time Comparison of L' ACCORD with zCHAFF'03 and zCHAFF'04

\begin{tabular}{||l|c||c||c|c|c||c||c|c||}
\hline & \multicolumn{3}{|c||}{} & zCHAFF'03 & L'ACCORD+zCHAFF'03 & zCHAFF'04 & L' ACCORD+zCHAFF'04 \\
\hline $\begin{array}{l}\text { Bench- } \\
\text { mark }\end{array}$ & $\begin{array}{c}\text { Vars } \\
\text { Clauses }\end{array}$ & $\begin{array}{c}\text { Solve } \\
(\mathrm{sec})\end{array}$ & $\begin{array}{c}\text { Var. } \\
\text { Time(s) }\end{array}$ & $\begin{array}{c}\text { Solve } \\
\text { Time(s) }\end{array}$ & $\begin{array}{c}\text { Total } \\
\text { Time(s) }\end{array}$ & $\begin{array}{c}\text { Solve } \\
\text { Time(s) }\end{array}$ & $\begin{array}{c}\text { Solve } \\
\text { Time(s) }\end{array}$ & $\begin{array}{c}\text { Total } \\
\text { Time(s) }\end{array}$ \\
\hline 4pipe & $5237 / 80213$ & 129 & 0.56 & 56.1 & 56.66 & 8.02 & 11.96 & 12.52 \\
\hline 5pipe & $9471 / 195452$ & 196.53 & 2.36 & 54.46 & 56.82 & 18.34 & 16.59 & 18.95 \\
\hline 4pipe_k & $5095 / 79489$ & 208.81 & 0.6 & 40.85 & 41.45 & 8.34 & 14.53 & 15.13 \\
\hline 5pipe_k & $9330 / 189109$ & 871.79 & 2.25 & 388.32 & 390.57 & 40.75 & 31.29 & 33.54 \\
\hline 4pipe_q0 & $5380 / 69072$ & 66.71 & 0.48 & 73.86 & 74.34 & 6.37 & 8.59 & 9.07 \\
\hline 5pipe_q0 & $10026 / 154409$ & 649.9 & 1.53 & 214.89 & 216.42 & 25.21 & 26.46 & 27.99 \\
\hline 6pipe & $15800 / 394739$ & $>2000$ & 6.53 & 827.06 & 833.59 & 132.82 & 144.72 & 151.25 \\
\hline 6pipe_k & $15346 / 408792$ & 105.52 & 8.76 & 141.45 & 150.21 & 71.49 & 57.64 & 66.4 \\
\hline 6pipe_q0 & $16775 / 315960$ & 104.48 & 4.88 & 82.44 & 87.32 & 43.37 & 46.65 & 51.53 \\
\hline 7pipe_q0 & $26512 / 536414$ & $>2000$ & 12.45 & 1523.16 & 1535.61 & 284.97 & 265.3 & 277.75 \\
\hline 8pipe_q0 & $39434 / 887706$ & $>2000$ & 31.16 & $>2000$ & - & 819.13 & 892.32 & 923.48 \\
\hline
\end{tabular}

\section{Conclusions and Future Work}

This paper has advocated the need to analyze constraint-variable relationships to derive an ordering of variables to guide SAT diagnosis. To analyze the tightness of the connectivity between variables, we have a proposed the degree of correlation as a qualitative and quantitative metric. Our technique models the constraint variable dependencies on a weighted graph and analyzes the graph's topological structure to derive the order. Our approach is fast, robust, scalable and can handle a large set of variables and constraints. The variable order derived by our procedure improves the performance of the solver by one or more orders of magnitude. As part of future work, we are exploring a dynamic variable order update strategy to be employed when conflict clauses are added to the database.

\section{References}

1. M. Davis and H. Putnam, "A Computing Procedure for Quantification Theory", Journal of the ACM, vol. 7, pp. 201-215, 1960.

2. M. Davis, G. Logemann, and D. Loveland, "A machine program for theorem proving", in Communications of the ACM, 5:394-397, 1962.

3. J. Marques-Silva and K. A. Sakallah, "GRASP - A New Search Algorithm for Satisfiability", in ICCAD'96, pp. 220-227, Nov. 1996. 
4. M. Moskewicz, C. Madigan, L. Zhao, and S. Malik, "CHAFF: Engineering and Efficient SAT Solver", in In Proc. Design Automation Conference, pp. 530-535, June 2001.

5. E. Goldberg and Y. Novikov, "BerkMin: A Fast and Robust Sat-Solver", in DATE, pp 142-149, 2002.

6. F. Aloul, A. Ramani, I. Markov, and K. Sakallah, "Solving Difficult SAT Instances in the Presence of Symmetry", in Proc. DAC, pp. 731-736, 2002.

7. J. Crawford, M. Ginsberg, E. M. Luks, and A. Roy, "Symmetry-Breaking Predicates for Search Problems", in Proceedings of the Fifth International Conference on Knowledge Representation and Reasoning (KR '96), 1996.

8. B. Selman, H. Kautz, and B. Cohen, "Local Search Strategies for Satisfiability Testing", DIMACS Series in Mathematics and Theoretical Computer Science, vol. 26, pp. 521-532, 1996.

9. M. Prasad, P. Chong, and K. Keutzer, "Why is ATPG Easy?", in Design automation Conf., pp. 22-28, June 1999.

10. F. Aloul, I. Markov, and K. Sakallah, "Mince: A static global variable-ordering for sat and bdd", in International Workshop on Logic and Synthesis. University of Michigan, June 2001.

11. F. Aloul and et al., "Satometer: How Much Have We Searched?", IEEE Trans. $C A D$, vol. 22, pp. 995-1004, 2003.

12. L. Zhang and S. Mailk, "Extracting Small Unsatisfiable Cores from Unsatisfiable Boolean Formula", in Sixth International Conference on Theory and Applications of Satisfiability Testing (SAT 2003), 2003.

13. E. Goldberg and Y. Novikov, "Verification of Proofs of Unsatisfiability for CNF Formulas", in Design, Automation and Test in Europe (DATE ), 2003.

14. S. Pilarski and G. Hu, "SAT with Partial Clauses and Backleaps", in In Proc. $D A C$, pp. 743-746, 2002.

15. R. Damiano and J. Kukula, "Checking Satisfiability of a Conjunction of BDDs", in DAC, pp 818-823, 2003.

16. P. Kalla, Z. Zeng, M. J. Ciesielski, and C. Huang, "A BDD-based Satisfiability Infrastructure using the Unate Recursive Paradigm", in Proc. Design Automation and Test in Europe, DATE'00, 2000.

17. J. P. M. Silva, "The Impact of Branching Heuristics in Propositional Satisfiability Algorithms", in Portuguese Conf. on Artificial Intelligence, 1999.

18. R. Dechter and J. Pearl, "Network-based Heuristics for Constraint-Satisfaction Problems", Artificial Intelligence, vol. 34, pp. 1-38, 1987.

19. E. Amir and S. McIlraith, "Partition-Based Logical Reasoning", in 7th International Conference on Principles of Knowledge Representation and Reasoning (KR'2000), 2000.

20. E. Amir and S. McIlraith, "Solving Satisfiability using Decomposition and the Most Constrained Subproblem", in LICS workshop on Theory and Applications of Satisfiability Testing (SAT 2001), 2001.

21. P. Bjesse, J. Kukula, R. Damiano, T. Stanion, and Y. Zhu, "Guiding SAT Diagnosis with Tree Decompositions", in Sixth International Conference on Theory and Applications of Satisfiability Testing (SAT 2003), 2003.

22. A. Gupta, Z. Yang, P. Ashar, L. Zhang, and S. Malik, "Partition-based Decision Heuristics for Image Computation using SAT and BDDs", in Proceedings of the 2001 IEEE/ACM international conference on Computer-aided design (ICCAD), pp. 286-292. IEEE Press, 2001.

23. E. Amir, "Efficient Approximation for Triangulation of Minimum Treewidth", in 17th Conference on Uncertainty in Artificial Intelligence (UAI '01), 2001. 
24. J. Huang and A. Darwiche, "A structure-based variable ordering heuristic for SAT", in Proceedings of the 18th International Joint Conference on Artificial Intelligence (IJCAI), pp. 1167-1172, August 2003.

25. A. Caldwell, A. Kahng, and I. Markov, "Improved Algorithms for Hypergraph Bipartitioning", in Proc. Asia-Pacific DAC, 2000.

26. F. A. Aloul, I. L. Markov, and K. A. Sakallah, "FORCE: A Fast and Easy-ToImplement Variable-Ordering Heuristic", in Proceedings of the 13th ACM Great Lakes symposium on VLSI, pp. 116-119, 2003.

27. V. Durairaj and P. Kalla, "Guiding CNF-SAT Search via Efficient Constraint Partitioning", in ICCAD, pp. 498 - 501, 2004.

28. V. Durairaj and P. Kalla, "Exploiting Hypergraph Partitioning for Efficient Boolean Satisfiability", in HLDVT, pp. 141-146, 2004.

29. V. Durairaj and P. Kalla, "Dynamic Analysis of Constraint-Variable Dependencies to Guide SAT Diagnosis", in HLDVT, pp. 135-141, 2004.

30. F. Aloul, I. Markov, and K. Sakallah, "MINCE", http://www.eecs.umich.edu/ faloul/Tools/mince/.

31. E. Amir and S. McIlraith, "The Partitioning and Reasoning Project", http://wwwfaculty.cs.uiuc.edu/ eyal/decomp/.

32. R. C. Prim, "Shortest connection networks and some generalizations", The Bell System Technical Journal, vol. 36, pp. 1389-1401, 1957.

33. N. Eén and N. Sörensson, "An Extensible SAT Solver", in 6th International Conference, SAT, 2003.

34. M. Velev, "Sat benchmarks for high-level microprocessor validation", http://www.cs.cmu.edu/ mvelev. 